\title{
Effect of kefir on Fusobacterium nucleatum in potentially preventing intestinal cancer
}

\author{
Zeynep Banu Guzel-Seydim*, Merve Dibekci, Ece Cagdas, and Atıf Can Seydim \\ ${ }^{1}$ Suleyman Demirel University Faculty of Engineering Department of Food Engineering, Isparta \\ 32260, Turkey
}

"Corresponding author: Zeynep Banu Guzel-Seydim, PhD, Professor, Suleyman Demirel University, Faculty of Engineering, Department of Food Engineering, Isparta 32260, Turkey

Submission Date: June 9, 2016, Accepted Date: July 26, 2016, Publication Date: July 30, 2016

\begin{abstract}
Background: Fusobacterium spp. are known to be part of mouth and intestinal microbiota. Fusobacterium nucleatum is an obligate anaerobe, Gram negative, non-spore forming pleomorphic bacillus that can cause diseases not only in the mouth and teeth but also in the brain, pleura, lungs and liver. It was noted that $F$. nucleatum induces fetal death (fetal demise) in pregnant women. Recent studies indicate that $F$. nucleatum could lead to colon cancer by binding to the epithelial tissue. Kefir is produced from kefir grains that are a source of probiotics. Fermented dairy products and especially kefir and yogurt are significant for functional nutrition. In kefir grains, lactic acid bacteria, acetic acid bacteria and yeasts are embedded in a polysaccharide matrix, called kefiran. When kefir grains are added to milk and incubated for approximately $22 \mathrm{~h}$ at $25^{\circ} \mathrm{C}$, microorganisms in the grains continue to proliferate in milk with the production of functional metabolic compounds. While yogurt has mainly two bacteria, authentic kefir has its characteristic Lactobacillus kefiranofaciens, Lactobacillus kefir and Lactobacillus kefirgranum, in addition to many other types of lactic acid bacteria (LAB). Previous studies have indicated that fermented dairy products can cause probiotic effects such as improvement in digestive system health, reduction in serum cholesterol, improvement in lactose tolerance, improvement in immune function, control of irritable bowel symptoms, and anticarcinogenic properties.
\end{abstract}

Objective: The aim of this research was to report the effects of fermented dairy products in vitro on the growth of F. nucleatum. Milk, kefir made from natural kefir grains, commercial kefir produced from kefir starter culture, yogurt produced from natural yogurt starter culture and commercial yogurt produced from yogurt starter culture were used against $F$. nucleatum.

Methods: F. nucleatum (ATCC 25586) was grown in Fluid Thioglycollate Medium at $37^{\circ} \mathrm{C}$ for 3 days under anaerobic incubation. Kefir was made from authentic kefir grains with $2 \%$ by volume inoculation at $25^{\circ} \mathrm{C}$ for $22 \mathrm{~h}$ fermentation. Yogurt was made using a natural starter culture. The inhibition effects of fermented dairy products were determined in vitro against $F$. nucleatum. Zone inhibition tests were conducted using sterile disks that were immersed into kefir and yogurt samples and subsequently placed on Brucella Blood Agar with Hemin and Vitamin K1 inoculated 
with F. nucleatum; plates were incubated at $37^{\circ} \mathrm{C}$ for 3 days under anaerobic conditions. Inhibition zones were determined after the incubation was completed. Additionally, lactic acid bacteria (counted on MRS and m17), yeast (PDA) and related pathogen were observed after anaerobic incubation by adding a certain amount of kefir and yogurt cultures into $F$. nucleatum inoculated medium.

Results: Authentic kefir samples exhibited distinct inhibitory zone against $F$. nucleatum after incubation agar plates. The largest zone of inhibition (with $9.5 \mathrm{~mm}$ ) was caused by natural kefir samples made from kefir grains. Yogurt samples provided $8.25 \mathrm{~mm}$ zone inhibition against $F$. nucleatum. The milk used to make kefir and yogurt did not cause any zone of inhibition. It was also discovered that number of $F$. nucleatum in Thioglicollate Medium decreased depending on inoculated concentrations of kefir and yogurt cultures.

Conclusion: Kefir is known to have positive effects on health and especially intestinal health. Therefore, these findings are important for showing an inhibition effect of fermented dairy products against a pathogen and possible carcinogen. These results suggest that regular consumption of natural fermented dairy products especially kefir should be included in a functional diet. The impact of these cultured dairy products could be promising and warrants further investigation with in vivo studies.

Keywords: Fusobacterium nucleatum, pathogen, carcinogen, fermented foods, kefir, kefir grains

\section{BACKGROUND}

Fusobacterium nucleatum has been known for a long time as a mouth pathogen. It is a spindleshaped, Gram negative, asporogenic, pleomorphic bacillum. The organism is considered a strict anaerobe, but there are studies demonstrating that it can live under in a $6 \%$ oxygen atmosphere [1]. The bacterium has a length between 5 and $10 \mu \mathrm{m}$ with a straight or bent posture. The cells are immobile and non-capsulated. One end of long bacilli is thick and the other is pointed. The thick ends come together to form a spindle shaped appearance. F. nucleatum fluoresces yellowish light green under UV light. Although generally non-hemolytic, F. nucleatum can be mildly hemolytic where they heavily propagate. In addition to mouth and gum diseases, this pathogen can cause brain, pleura, lung and liver abscesses, and may cause Vincent angina, Lemirre disease, Noma disease, ulcerative somatitis and gingivitis in cooperation with other bacteria [2]. Recent studies show that in patients with poor oral health, F. nucleatum may pass through the placenta in women and cause still births [3].

Colorectal cancers are one of the most significant causes of mortality and morbidity in cancer.

Worldwide, it is the third most common cancer type in women and fourth for men. Colon cancer is ranked right after lung cancer in cancer-based deaths in the USA, Canada and almost all European countries [4]. Moreover, the bacteria can attach itself to the epithelium surface with a unique special protein structure, and stress the cell from the attachment point to trigger a mutant cell division. This situation encourages the formation of ulcerative colitis and cancer tissues in intestinal tissues. This attachment also acts as a bridge that allows other pathogens to attach to the 
surface and may cause co-aggregation. Consequently, $F$. nucleatum is associated with intestinal diseases and especially colon cancer. In recent studies, it was observed that colonies of $F$. nucleatum were present in areas near colon cancer tissues in human colon biopsies $[5,6]$.

Kefir and yogurt are among the probiotic fermented milk products that have many functional properties. Natural kefir is prepared with "kefir grains," which are sources of probiotic microorganisms which proliferate in the product during fermentation at $25^{\circ} \mathrm{C}$ for 22 hours. The most important parameter in kefir production is starter culture selection. The characteristic kefir bacteria Lactobacillus kefiranofaciens, L. kefir, L. kefirgranum and yeasts are not found in commercial kefir starter cultures used around the world. Commercial kefir is made from selected lactic acid bacterial strains and not from kefir grains. In kefir grains, the polysaccharide known as kefiran is located inside the unique grain matrix that is produced through simultaneous lactic acid and ethyl alcohol fermentation. The product obtained by fermenting milk with kefir grains at $25^{\circ} \mathrm{C}$ for 22 hours has many unique beneficial metabolites. Previous studies have indicated that kefir has many functional properties such as protecting and healing the gut, maintaining mouth and tooth health through kefir metabolites and unique microflora, supporting the treatment in digestive disorders, lowering cholesterol, and possessing potent antimicrobial and anti-tumor properties [7].

In the intestine, a colony of bacteria must be able to adhere to the epithelium surface to be able to propagate [8]. It is known that probiotics limit the colonization opportunities for other bacteria since they compete for adherence [9]. Kefir has antimicrobial properties against some bacteria groups due to its competitive flora and unique metabolites. It is reported that kefir inhibits the bacterial growth of $S$. aureus [10, 11], K. pneumoniae, E. coli [12], Listeria monocytogenes, Salmonella typhimurium, S. enteriditis, S. flexneri, Yersinia enterocolitica [9, 13], and E. coli O157:H7.

This study attempts to investigate the possible inhibition effect of fermented dairy products such as the probiotic-rich kefir and probiotic yogurt against $F$. nucleatum, which causes pathogenic and tumorogenic activities by adhering to the colon epithelium in a manner similar to probiotics. Kefir is well known for its unique microflora that has many functional properties. This study is expected to define a new indirect functional property of probiotics against colon cancer and to increase the awareness of the importance of probiotics in human nutrition.

\section{METHODS}

\section{Materials}

Natural kefir grains (Büyüdanem) and probiotic yogurt culture (Büyüyo) (Danem Ltd., Süleyman Demirel University Technopark, Isparta, Turkey) were used to produce natural kefir and probiotic yogurt. For kefir and yogurt, reconstituted milk with $12 \%$ dry matter was prepared from dried milk and pasteurized at $85^{\circ} \mathrm{C}$ for 15 minutes. Commercial kefir and yogurt samples were obtained from grocery stores in Isparta, Turkey. A pure culture of lyophilized Fusobacterium nucleatum subsp. nucleatum $\left(\mathrm{ATCC}^{\circledR} 25586^{\mathrm{TM}}\right.$, UK) strain was obtained as a lyfo disc and used after being activated in Thioglycollate Fluid Medium (TFM, BD- 211260, Difco ${ }^{\mathrm{TM}}$, New Jersey, USA). The culture was spread plated on Brucella Blood Agar (BBA, BD Difco ${ }^{\text {TM }}$, New Jersey, USA) with hemin and vitamin K1 (5\% defibrinated sheep blood). The pour plate method was used with Lactobacillus 
MRS agar (MRS, Merck 1.10660, Darmstadt, Germany) and M17 Agar (Merck 115108, Darmstadt, Germany) to count lactic acid bacteria and with potato dextrose agar (PDA, Merck 110130, Darmstadt, Germany) to count yeasts. TFM was used to activate pure F. nucleatum cultures and to regularly transfer the culture. Peptone water (buffered, Merck 146596 Darmstadt, Germany) was used to prepare the dilutions. Sterile blank discs (Bioanalyse ${ }^{\circledR}$, Ankara, Turkey) were used for the disc diffusion method. The Anaerocult ${ }^{\circledR}$ system (Merck-113829 Anaerocult ${ }^{\circledR}$ A, Merck-116387 Anaerobic jar 2.5 L, Merck-115112 Anaerotest ${ }^{\circledR}$, Darmstadt, Germany) was used to create anaerobic conditions.

\section{Kefir Fermentation in the Presence of $\boldsymbol{F}$. nucleatum and Microbiological Analyses}

The study aims to monitor the changes during kefir fermentationof milk in the presence of $F$. nucleatum under the optimal growth conditions for $F$. nucleatum (anaerobic at $37^{\circ} \mathrm{C}$ ). Kefir samples without $F$. nucleatum were produced under both aerobic and anaerobic environments as control groups. A natural kefir culture obtained from incubating kefir grains in reconstituted milk (as above) at $25{ }^{\circ} \mathrm{C}$ for $18-24$ hours was used as an inoculum into an activated culture of $F$. nucleatum (in TFM broth) at a rate of $2 \% \mathrm{v} / \mathrm{v}$. A natural probiotic yogurt was similarly inoculated into a culture of $F$. nucleatum (in TFM broth) as well as a commercial kefir and a commercial yogurt product. After 24 hours of anaerobic incubation at $37^{\circ} \mathrm{C}$, samples were examined by dilution plating on MRS, M17 and PDA media via the pour plate method, and by spread plating on BBA media directly from the sample. MRS and M17 media were incubated for 3 days at $37^{\circ} \mathrm{C}$, BBA media for 3 days at $37^{\circ} \mathrm{C}$ and PDA medium for 5 days at $25^{\circ} \mathrm{C}$. Countable plates were considered those with 30 to 300 colonies. The study was carried out in two repetitions.

A disk diffusion method was used to determine the potential inhibition of $F$. nucleatum by natural kefir, probiotic yogurt, commercial kefir and commercial yogurt samples. Sterile blank discs were soaked with these fermented dairy product samples $(0.25 \mathrm{ml})$ and placed over BBA surfaces containing $5 \log F$. nucleatum which had been prepared by plating a liquid culture adjusted using a spectrophotometer. These samples were incubated under anaerobic conditions for 3 days at $37^{\circ} \mathrm{C}$ and the inhibition zones that formed in the Petri dishes were measured.

\section{Verification Tests}

Basic verification tests were carried out to differentiate lactic acid bacteria colonies from $F$. nucleatum colonies. In BBA medium, both lactic acid bacteria and $F$. nucleatum colonies can be observed. To properly identify these colonies, gram staining was performed. Thus, gram-positive lactic acid bacteria were easily separated from gram-negative $F$. nucleatum. The colonies formed in BBA by F. nucleatum are also known to fluorescence under UV light.

\section{RESULTS AND DISCUSSION}

Microbiological Results after Kefir Fermentation in the Presence of $\boldsymbol{F}$. nucleatum After 2\% kefir culture and $2 \%$ pure $F$. nucleatum culture were first grown in broth and then added to 
reconstituted milk and incubated at $37^{\circ} \mathrm{C}$ under aerobic and anaerobic conditions, lactic acid bacteria yeasts and F. nucleatum were enumerated (Figure 1).

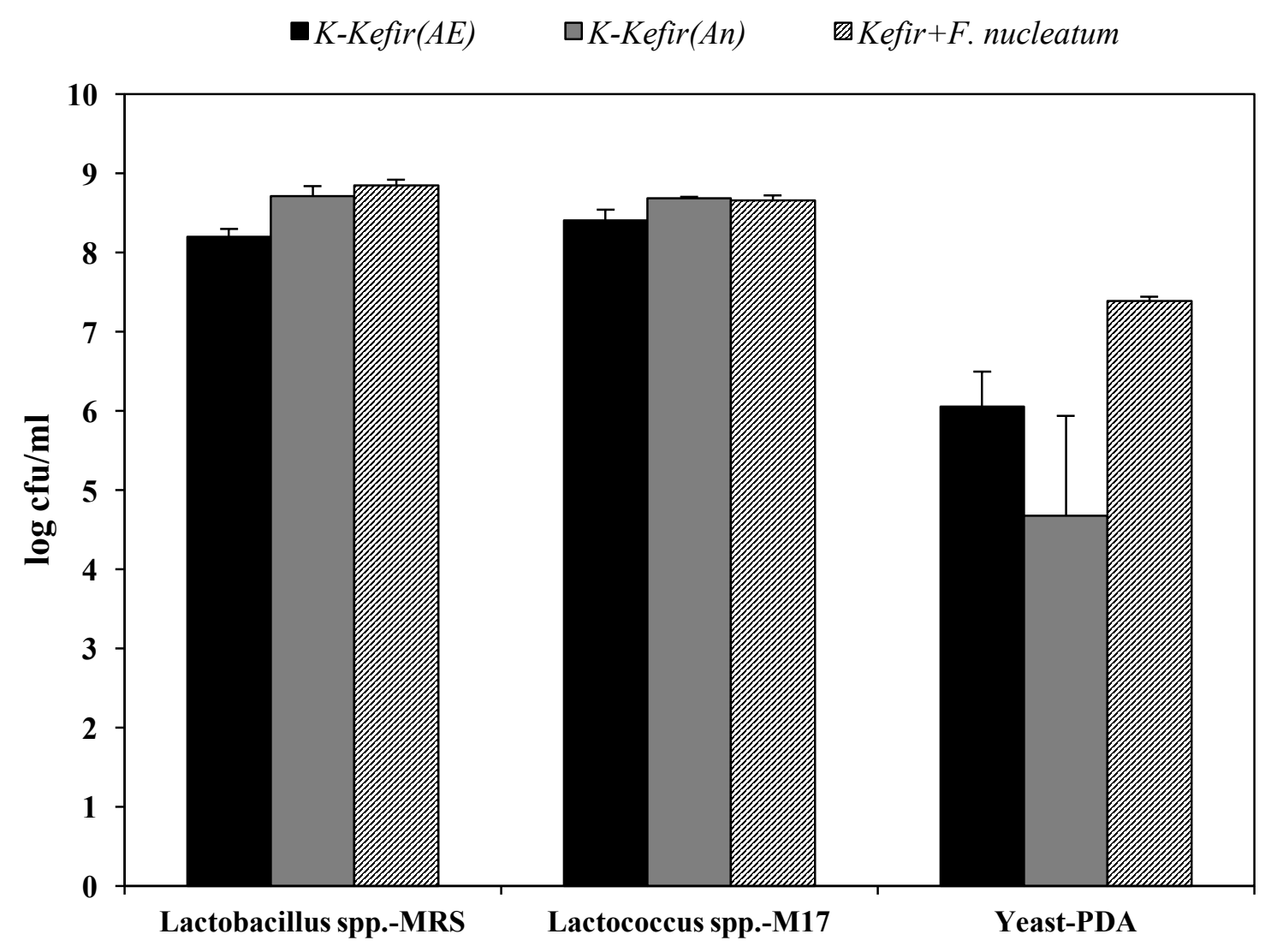

Figure 1. Microbiological analysis results after kefir fermentation in the presence of $F$. nucleatum

K-Kefir (Ae): Kefir made with 2\% natural kefir culture added to pasteurized milk under aerobic conditions. K-Kefir(An): Kefir made with $2 \%$ natural kefir culture added to pasteurized milk under anaerobic conditions.

Kefir + F. nucleatum (An): Kefir made with $2 \%$ natural kefir culture and the same amount of $F$.nucleatum added to pasteurized milk under anaerobic conditions.

Fermentation occurred in the medium with $F$. nucleatum. Although optimal conditions for fermentation could not be provided and the temperature was higher than the ideal kefir fermentation temperature, the number of lactic acid bacteria and yeast in the kefir inoculated with $F$. nucleatum were higher than in both the anaerobic and aerobic control groups. As the study was carried out in an anaerobic environment which should suppress the kefir culture, the number of yeasts in the anaerobic conditions where $F$. nucleatum was present were higher than the number of yeasts in normal, aerobic conditions. Therefore, a stronger fermented milk clot was observed in $F$. nucleatum added kefir samples; the reason could be related to lactic acid bacteria that increased the acidity or the increased amount of yeasts (Figure 1) may be provided more proteolytic enzymes, or F. nucleatum may also have proteolytic enzymes. The fact that an increase in the number of 
lactic acid bacteria and especially yeasts after adding $F$. nucleatum may suggest a development of defense or inhibition mechanism via bacteriocins, organic acids or other metabolites against the pathogen.

The same samples were examined to determine whether the number of $F$. nucleatum decreased in BBA. Pure culture of $F$. nucleatum was spread plated on BBA, resulting in green, viscous $F$. nucleatum colonies. When the mixed culture of kefir inoculated with $F$. nucleatum was similarly plated, no $F$. nucleatum colonies were detected.

\section{Disc Diffusion-Zone Inhibition Results}

To determine the potential inhibition of $F$. nucleatum by natural kefir, probiotic yogurt, commercial yogurt and commercial kefir samples, sterile filter paper discs were soaked with those samples and deposited on $F$. nucleatum spread-plated samples. The cell density of the $F$. nucleatum culture was adjusted using a spectrophotometer prior to spread plating on BBA surfaces. These samples were incubated in anaerobic conditions for 3 days at $37^{\circ} \mathrm{C}$ and the zones that formed in the petri dish were observed (Figure 2).

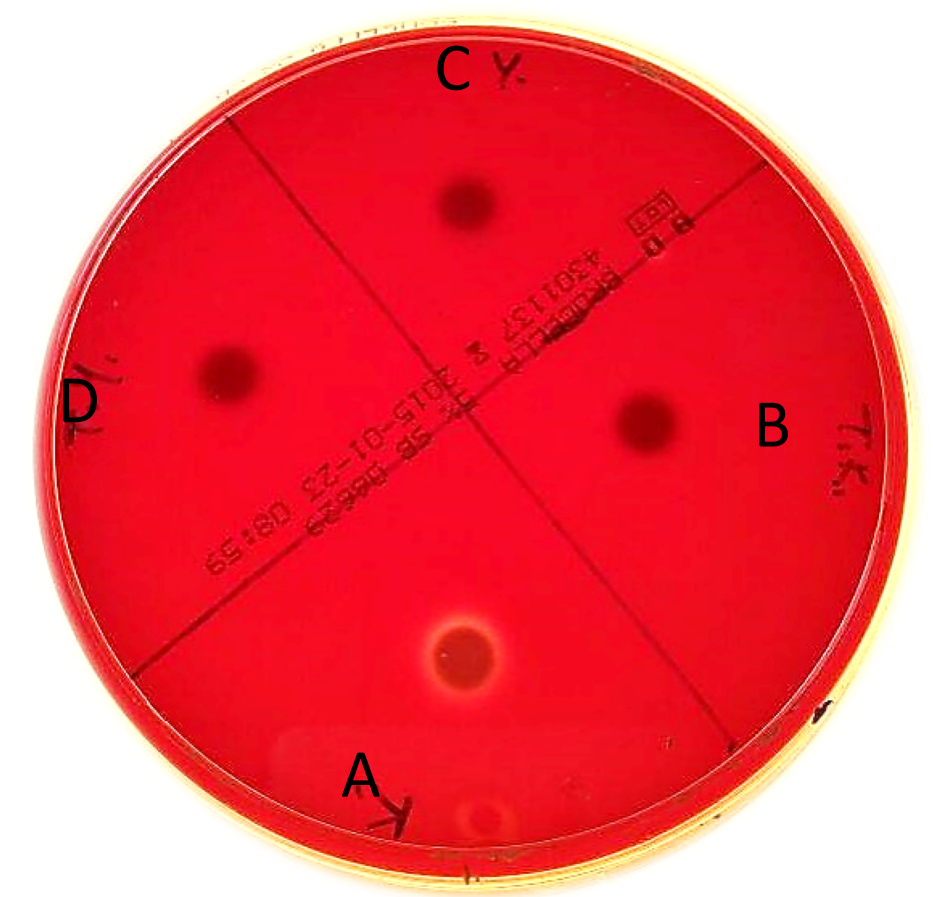

Figure 2. F. nucleatum inhibitory zone forming areas on discs soaked with Natural Kefir (A), Commercial Kefir (B), Probiotic Yogurt (C) and Commercial Yogurt (D) $\left(37^{\circ} \mathrm{C}, 4\right.$ days incubation, Media: Brucella Blood Agar with Hemin and Vitamin K1- Anaerobic Conditions 


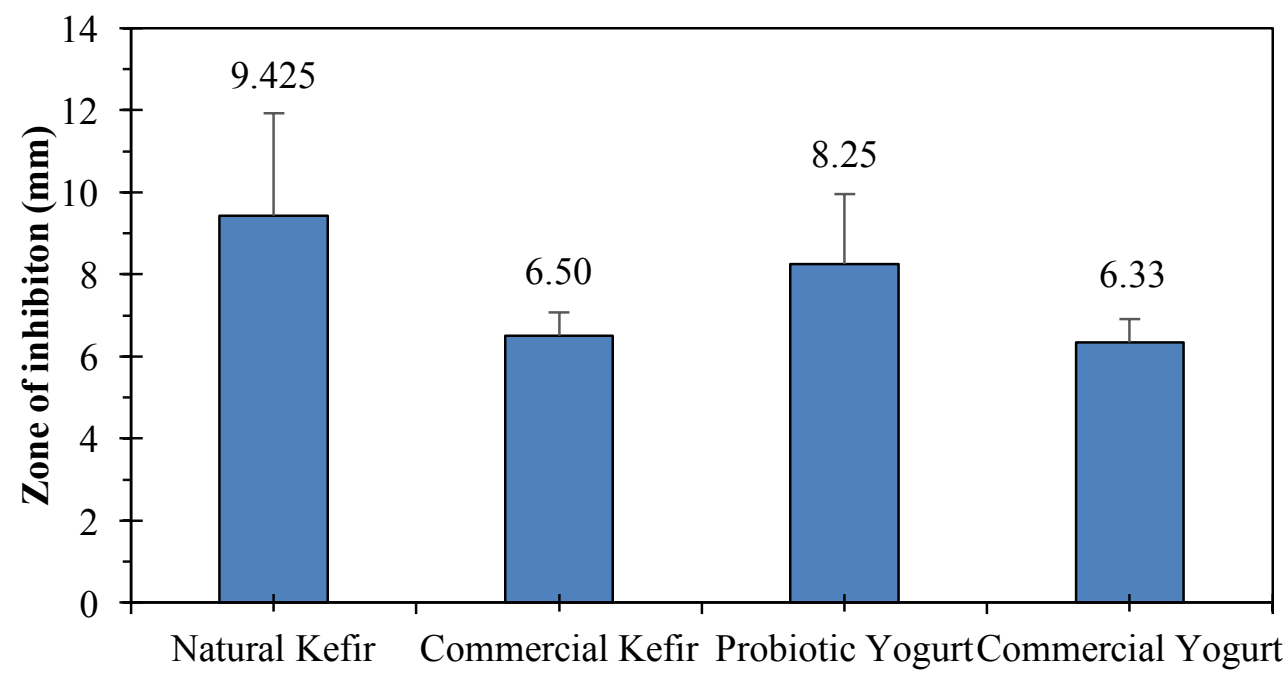

Figure 3. F. nucleatum inhibitory zones (mm)

The largest and the most obvious zone diameters were observed around the disc soaked with natural kefir. This was followed by probiotic yogurt, commercial kefir and commercial yogurt. Commercial kefirs contain only limited cultures and do not contain characteristic kefir microflora. Health aspects of consumed kefir greatly depend on the quality of kefir culture authenticity. The results support the hypothesis that fermented dairy products, especially natural kefir and probiotic yogurt, may have F. nucleatum inhibitory characteristics. Their potent antimicrobial and antitumor properties have also reported in previous studies [7, 13, 22].

During the study, samples were taken from colonies and gram-stained for identification of colonies on BBA. Thus, gram-negative F. nucleatum colonies could be separated from grampositive lactic acid bacteria colonies. Additionally, TFM media containing pure culture were also gram-stained in various stages of the study to assess the purity and viability of the culture and the existence of contaminants. In some stages of the study, the density of $F$. nucleatum colonies on the BBA agar surface were assessed using a UV lamp. It was observed that as the amount of kefir and probiotic yogurt increased, the density of $F$. nucleatum colonies that fluoresced under UV light decreased.

\section{CONCLUSION}

Colon cancer is a type of cancer that is very common today. These results suggest that milk, and fermented dairy products, especially kefir, have inhibitory effects against the bacterial pathogen F. nucleatum. Milk contains calcium, proteins and carbohydrates that promote the propagation of probiotics and their adhesion to the colon, while Kefir contains colon-adhering lactic acid bacteria and unique yeast flora. $F$. nucleatum is known to cause colon cancer by adhering to the colon epithelium and causing cell mutation. L. kefiri, L. kefiranofaciens and L. kefirgranum, which were proven by recent studies to have strong probiotic properties and positive health effects, are only present in kefir grains [14, 15, 16, 17, 18]. Moreover, the Saccharomyces and Kluyveromyces yeast strains unique to kefir grains also have important probiotic properties and antimicrobial effects that were observed in recent studies [19, 20, 21]. 
The reason for commercial kefir having a lower observed inhibitory effect is generally thought to be due to the fact that the starter culture microflora is very different from the original in commercial kefir. In most cases, commercial kefir contains fewer lactic acid bacteria strains and no yeast. These cultures and the yeast are very important parameters in kefir studies.

The lactic acid bacteria in natural kefir are known to have antimicrobial effects against pathogenic bacteria and to have anti-tumor effects, cholesterol lowering effects, effects that strengthen the immune system, and effects against gastrointestinal system disorders $(22,23,24)$. Results of this study demonstrated that natural kefir produced from kefir grains inhibited/suppressed the activity of the $F$. nucleatum, which is directly correlated with cancer and which has been associated with mouth disease for many years. As kefir is a natural fermented, drinkable dairy product, it potentially also has benefits for oral health. As F. nucleatum first colonizes the mouth and is carried to other organs in the body through the bloodstream to adversely affect the health, it may be possible to inhibit the bacteria even in the mouth with drinking kefir. The study is a pioneering study in this area and advanced in vivo studies are necessary to continue this work.

Competing Interests: The authors have no financial interests or conflicts of interest.

Author's Contributions: ZBGS, ACS and MD designed the research; MV, ZBGS and EC performed the research; MD did bacterial cultures and plating; ZBGS and EC prepared the kefir grains and kefir samples, yogurt starter culture and yogurt samples; MD and EC analyzed the data; MD, ZBGS, ACS, EC prepared the manuscript, figures, references.

Acknowledgements and Funding: This research was partially supported by TUBITAK (2229). We thank to Danem Inc. (Lake Districts Technopark, Suleyman Demirel University, Isparta) for kindly providing natural kefir grains and probiotic yogurt starter culture.

\section{REFERENCES}

1. WEC Moore, LVR Holdeman, W Kelley: Genus II. Fusobacterium Knorr 1922, 4AL, In NR Krieg and J.G Holt (eds), Bergey's manual of systematic bacteriology. Williams and Wilkins Baltimore; 1984, 631-637.

2. AI Bolstad, HB Jensen, V Bakken: Taxonomy, Biology, and Periodontal Aspects of Fusobacterium nucleatum. Clin Microbiol Rev 1996, 55-71.

3. YW Han, et al.: Term stillbirth caused by oral Fusobacterium nucleatum. Obstet Gynecol 2010, 115: 442-445.

4. RS Bresalier, M Feldman, LS Freidman, MH Sleisenger: Malignant neoplasms of the large intestine Gastrointestinal and liver disease, pathophysiology, diagnosis, management. Small and large intestine: Philadelphia Saunders; 2002, 2215-62.

5. M Castellarin, et al.: Fusobacterium nucleatum infection is prevalent in human colorectal carcinoma. Genome Res 2011, 22: 299-306.

6. M Nisha, X Wu, L Wang: Management of peri-implantitis: a systematic review, 20102015. Springer Plus 2016, 5: 105 (accepted, in print-December, 2016). 
7. T Kok-Tas: 2010. Kontrollü atmosfer uygulamasının kefir danesi ve kefir üzerine etkilerinin belirlenmesi. Suleyman Demirel University, Institute of Science-Dissertation.

8. RJ Gibbons:. Bacterial adhesion to oral tissues: A model for infectious diseases. J Dent Res 1989, 68(5): 750-760.

9. A Santos, et al.: The antimicrobial properties of different strains of Lactobacillus spp. isolated from kefir. Syst Appl Microbiol 2003, 26: 434-437.

10. ZN Yuksekdag, Y Beyath, B Aslim: Metabolic activities of Lactobacillus spp. strains isolated from kefir. Nahrung 2004, 48: 218-220.

11. K Rodrigues, et al.: Antimicrobial and healing activity of kefir and kefiran extract. Int $\mathbf{J}$ Antimicrob Ag 2005, 25: 404-408.

12. T Serot, X Dousset, J Zucca, N Torcatis: Mise en e'vidence et purification partielle de substances antibacteriennes produites par Leuconostoc mesenteroides and Lactobacillus plantarum isoles de grains de kefyr. Microbiol. Aliment Nutr 1990, 8: 71-76.

13. M Gulmez, A Guven: Survival of Escherichia coli 0157:H7, Listeria monocytogenes 4b and Yersinia enterocolitica 03 in different yoghurt and kefir combinations as prefermentation contaminant. J Appl Microb 2003, 95: 631-636.

14. P Carasi, et al.: Impact of Kefir Derived Lactobacillus kefiri on the Mucosal Immune Response and Gut Microbiota. J Immunol Res 2015, 1-12.

15. SY Wang, et al: Investigation of microorganisms involved in biosynthesis of the kefir grain. Food Microbiol 2012, 32(2): 274-85.

16. SY Chen, MJ Chen: Effects of Lactobacillus kefiranofaciens M1 isolated from kefir grains on germ-free mice. PLOS One 2013, 8(11): e78789.

17. MC Franco, et al.: Administration of kefir-fermented milk protects mice against Giardia intestinalis infection. J Med Microb 2013, 62(Pt 12): 1815-22.

18. M Ghoenum, J Gimzewski: Apoptotic effect of a novel kefir product, PFT, on multidrugresistant myeloid leukemia cells via a hole-piercing mechanism. Int J Oncol 2014, 44(3): 830-7.

19. D Romanin, et al.: Down-regulation of intestinal epithelial innate response by probiotic yeasts isolated from kefir. Int J Food Microbiol 2010, 140(2-3): 102-8.

20. G Diosma, et al.: Yeasts from kefir grains: isolation, identification, and probiotic characterization. World J Microb Biot 2014, 30(1): 43-53.

21. FS Erdogan, T Kok Tas: Süt ürünlerinden izole edilen mayaların probiyotik özellikleri. 2. İç Anadolu Bölgesi Tarım ve Gıda Kongresi, Nevsehir; 2015, 171.

22. ZB Guzel-Seydim, T Kok-Tas, AK Greene: Review: Functional Properties of Kefir. Critical Reviews in Food Sci Nutr 2011, 51(3): 261-268.

23. Z Guzel-Seydim, T Kok-Tas, AK Greene: Kefir and koumiss: Microbiology and Technology. In Development and Manufacture of Yogurt and Other Functional Dairy Products: CRC Press; 2010, 143-164.

24. ZB Guzel-Seydim, AC Seydim, AK Grenee, T Tas: Determination of Antimutagenic Properties of Some Fermented Milks Including Changes in the Total Fatty Acid Profiles including CLA. Int J Dairy Technology 2006, 59 (3): 209-215. 